\title{
Propriétés arithmétiques d'une série liée aux fonctions thêta
}

par

\author{
Daniel Duverney (Lille)
}

1. Introduction. Soit $q \in \mathbb{Z},|q| \geq 2$. Nous définissons

$$
x_{q}=\sum_{n=0}^{\infty} q^{-n^{2}} .
$$

Il est clair que $x_{q}$ est un nombre irrationnel, puisque son développement $q$-adique est formé d'une suite non périodique de 0 et de 1 .

J. Liouville a démontré l'irrationalité de $x_{q}$ en l'approximant par les sommes partielles de la série qui le définit [14].

L'étude des propriétés arithmétiques de $x_{q}$ s'est poursuivie grâce à l'introduction de la fonction

$$
f(z)=\sum_{n=0}^{\infty} q^{-n(n+1) / 2} z^{n} .
$$

On peut citer, sur ce sujet, les travaux de L. Tschakaloff [18], de P. Bundschuh [2], de P. Bundschuh et M. Waldschmidt [4]. Pour une bibliographie plus complète sur ce sujet, on pourra consulter [3].

En particulier, P. Bundschuh dans [2] a démontré que $x_{q}$ n'est pas un nombre de Liouville.

Très récemment, $\mathrm{P}$. Borwein a prouvé dans [1] que

$$
\sum_{n=1}^{\infty} \frac{(-1)^{n}}{q^{n}+r} \notin \mathbb{Q} \quad \text { si } r \in \mathbb{Q}, r \neq-q^{n} .
$$

On en déduit que $x_{q}^{2}-x_{q}$ est irrationnel ([12], Th. 312, p. 258).

Les nombres $x_{q}$ se trouvent liés de manière très simple à la fonction $\theta_{3}$ de Jacobi, puisque l'on a ([5], p. 65)

$$
2 x_{q}=1+\theta_{3}\left(0, \frac{\log q}{i \pi}\right) .
$$

Le but de ce travail est de démontrer le résultat suivant : 
ThÉORÈme 1. Soit $q \in \mathbb{Z},|q| \geq 2$. Alors

$$
x_{q}=\sum_{n=0}^{\infty} q^{-n^{2}} \quad \text { est non quadratique. }
$$

P. Erdős conjecture par ailleurs dans [7] que, si $n_{k}$ est une suite d'entiers vérifiant $n_{k}>c k^{2}$, alors $\sum_{k=1}^{\infty} q^{-n_{k}}$ est non quadratique. Cette conjecture pourrait peut-être se démontrer par une méthode analogue à celle utilisée ici, bien que cela paraisse, a priori, beaucoup plus difficile.

Pour démontrer le théorème 1 , nous utiliserons le critère d'irrationalité suivant :

ThÉORÈme 2. Soit $q \in \mathbb{Z},|q| \geq 2$. Soit $(a(n))_{n \in \mathbb{N}}$ une suite dans $\mathbb{Z}^{\mathbb{N}}$ vérifiant les propriétés suivantes :

(a) $a(n) \neq 0$ pour une infinité de valeurs de $n$.

(b) Pour $n$ assez grand, $|a(n)| \leq r(n)$, avec

$\left(\mathrm{b}_{1}\right) r(n)>0$,

$\left(\mathrm{b}_{2}\right) \lim \sup r(n+1) / r(n)<|q|$.

(c) Il existe une infinité d'entiers $k \in \mathbb{N}$, et des entiers $n_{k} \in \mathbb{N}$, tels que :

$\left(c_{1}\right) a\left(n_{k}+1\right)=a\left(n_{k}+2\right)=\ldots=a\left(n_{k}+k\right)=0$,

$\left(\mathrm{c}_{2}\right) \lim _{k \rightarrow \infty} r\left(n_{k}+k+1\right) /|q|^{k}=0$.

Soit $x=\sum_{n=0}^{\infty} a(n) q^{-n}$. Alors si $x=\alpha / \beta \in \mathbb{Q}$, on a pour $k$ assez grand,

$$
\alpha q^{n_{k}}-\beta \sum_{n=0}^{n_{k}} a(n) q^{n_{k}-n}=0 .
$$

La démonstration du théorème 2 sera donnée au paragraphe 2 . Remarquons seulement que ce critère est basé tout simplement sur l'approximation de $x$ par ses sommes partielles et nous n'introduisons, par conséquent, aucun outil nouveau.

Il existe d'autres résultats d'irrationalité pour les séries $x=\sum_{n=0}^{\infty} a(n) q^{-n}$ comportant beaucoup de termes nuls. On peut citer les deux théorèmes suivants :

ThÉORÈme $3([10])$. Si $a(n) \in \mathbb{N}$ pour tout $n \geq 1$, et si $a(n)$ est non nul pour une infinité de valeurs de $n$, avec

$$
\sum_{k=1}^{n} a(k)=o(n),
$$

alors $\sum_{n=1}^{\infty} a(n) q^{-n}$ est irrationnel pour tout entier $q \geq 2$.

ThÉORÈme $4([6])$. Soit a $(1)<a(2)<\ldots$ une suite d'entiers satisfaisant $\lim _{n \rightarrow \infty}[a(n+1)-a(n)]=\infty$. Alors $\sum_{n=1}^{\infty} a(n) q^{-a(n)}$ est irrationnel pour tout entier $q \geq 2$. 
Aucun de ces deux théorèmes ne peut s'appliquer au cas qui nous occupe, même pour $q \geq 2$. Supposons, en effet, que $x_{q}$ soit quadratique, c'est-à-dire qu'il existe $A, B, C \in \mathbb{Z}, A \neq 0$, tels que

$$
A x_{q}^{2}+B x_{q}+C=0 .
$$

Ecrivons $x_{q}=\sum_{n=0}^{\infty} l(n) q^{-n}$ avec $l(n)=1$ si $n$ est le carré d'un entier naturel, $l(n)=0$ sinon. Alors

$$
x_{q}^{2}=\sum_{n=0}^{\infty} \sum_{k=0}^{n} l(k) l(n-k) q^{-n} .
$$

Des considérations bien connues ([12], p. 258 ; [8], p. 219) montrent que

$$
x_{q}^{2}=\sum_{n=0}^{\infty} \frac{\varrho(n)}{q^{n}}
$$

où $\varrho(n)$ désigne le nombre de décompositions de $n$ en somme des carrés de deux entiers positifs.

Alors (6) devient

$$
\sum_{n=0}^{\infty} \frac{A \varrho(n)+B l(n)}{q^{n}}+C=0 .
$$

On se ramène donc à prouver l'irrationalité de $\sum_{n=0}^{\infty} a(n) q^{-n}$, avec $a(n)=A \varrho(n)+B l(n)$. Puisque $a(n)$ peut changer de signe, ni le théorème 3 ni le théorème 4 ne peuvent être utilisés. Ils ne peuvent même pas permettre de démontrer que $x_{q}^{2}$ est irrationnel. C'est clair pour le théorème 4; en ce qui concerne le théorème 3 , on sait que ([12], p. 270, th. 339)

$$
\lim _{n \rightarrow \infty} \frac{\varrho(1)+\varrho(2)+\ldots+\varrho(n)}{n}=\frac{\pi}{4} .
$$

La condition (5) n'est donc pas vérifiée.

Il nous faudra, pour appliquer le théorème 2, un lemme assez fin sur la répartition des zéros de la fonction $\varrho(n)$. Ce lemme sera énoncé et démontré au paragraphe 4 . Sa démonstration utilise de manière essentielle le résultat suivant, dû à Yu. Linnik [13] :

ThÉORÈme 5. Soit $u_{n}=\zeta n+\chi$, avec $\zeta, \chi \in \mathbb{N}$, $\zeta$ et $\chi$ premiers entre eux, et $0<\chi<\zeta$. Soit $p(\zeta, \chi)$ le plus petit nombre premier de la suite arithmétique $u_{n}$. Il existe une constante absolue $L$ (constante de Linnik) telle que

$$
p(\zeta, \chi) \leq \zeta^{L}
$$

pour $\zeta$ assez grand.

La constante $L$ est effectivement calculable. On sait par exemple que $L \leq 20$ ([11]). Mais nous n'aurons pas besoin de cette estimation. 
Le plan de l'article est le suivant : dans le paragraphe 2, nous démontrons le théorème 2 . Dans le paragraphe 3 , nous donnons un exemple pédagogique d'application du théorème 2. Cet exemple nous permettra de motiver la forme générale du lemme technique sur les zéros de $\varrho(n)$. L'énoncé et la longue démonstration de ce lemme occuperont le paragraphe 4. Dans le paragraphe 5 enfin, nous démontrerons le théorème 1 .

2. Démonstration du théorème 2. Si $\beta x-\alpha=0$, avec $\alpha, \beta \in \mathbb{Z}$, alors

$$
\alpha q^{n_{k}}-\beta \sum_{n=0}^{n_{k}} a(n) q^{n_{k}-n}=\beta q^{n_{k}} \sum_{n=n_{k}+1}^{\infty} \frac{a(n)}{q^{n}} .
$$

D'où, en vertu de $\left(\mathrm{c}_{1}\right)$,

$$
\alpha q^{n_{k}}-\beta \sum_{n=0}^{n_{k}} a(n) q^{n_{k}-n}=\beta q^{n_{k}} \sum_{n=n_{k}+k+1}^{\infty} \frac{a(n)}{q^{n}} .
$$

Utilisant (b), nous obtenons

$$
\left|\alpha q^{n_{k}}-\beta \sum_{n=0}^{n_{k}} a(n) q^{n_{k}-n}\right| \leq|\beta||q|^{n_{k}} \sum_{n=n_{k}+k+1}^{\infty} \frac{r(n)}{|q|^{n}} .
$$

D'après l'hypothèse $\left(\mathrm{b}_{2}\right)$, on peut trouver $\left.\eta \in\right] 0,|q|[$ tel que $r(n+1) / r(n)$ $\leq \eta$ pour $n$ assez grand. Mais $n_{k}$ tend vers l'infini avec $k$ en vertu de (a), donc pour $k$ assez grand,

$$
\begin{aligned}
\left|\alpha q^{n_{k}}-\beta \sum_{n=0}^{n_{k}} a(n) q^{n_{k}-n}\right| & \leq|\beta||q|^{n_{k}} \sum_{n=n_{k}+k+1}^{\infty} \frac{r\left(n_{k}+k+1\right) \eta^{n-\left(n_{k}+k+1\right)}}{|q|^{n}} \\
& \leq|\beta||q|^{n_{k}} \frac{r\left(n_{k}+k+1\right)}{|q|^{n_{k}+k+1}} \sum_{n=0}^{\infty}\left(\frac{\eta}{|q|}\right)^{n} \\
& \leq \frac{|\beta|}{|q|-\eta} \frac{r\left(n_{k}+k+1\right)}{|q|^{k}} .
\end{aligned}
$$

Pour $k$ assez grand, l'hypothèse $\left(\mathrm{c}_{2}\right)$ et le fait que le membre de gauche de cette inégalité est entier entraînent que

$$
\alpha q^{n_{k}}-\beta \sum_{n=0}^{n_{k}} a(n) q^{n_{k}-n}=0 .
$$

Remarque. Dans le cas où tous les termes de la série sont positifs, c'est-à-dire $q \geq 2$ et $a(n) \geq 0, \forall n \in \mathbb{N}$, on peut conclure que $x$ est irrationnel (il suffit de reporter le résultat obtenu dans l'égalité (11)). Mais dans le cas général, on ne peut espérer qu'une contradiction de type arithmétique, 
faisant intervenir la notion de divisibilité. C'est ce qui apparaîtra nettement dans l'exemple simple que nous étudions maintenant.

\section{Un exemple pédagogique}

ThÉORÈme 6 . Soit $\left(p_{n}\right)_{n \in \mathbb{N}^{*}}$ la suite des nombres premiers de $\mathbb{N}$. Soit $q \in \mathbb{Z},|q| \geq 2$. Alors

$$
x=\sum_{n=1}^{\infty} p_{n} q^{-p_{n}} \quad \text { est irrationnel. }
$$

Dém onstration. Avant de démontrer ce théorème, remarquons qu'il ne peut se déduire du théorème 4 , puisque l'on ne connait pas $\lim \inf \left(p_{n+1}-\right.$ $p_{n}$ ) (voir par exemple [16], p. 191 et suivantes). Il ne peut non plus se déduire du théorème 3 , même si $q>0$, car si nous posons

$$
x=\sum_{n=0}^{\infty} a(n) q^{-n}
$$

avec $a(n)=n$ si $n$ est premier et $a(n)=0$ si $n$ est composé, nous avons

$$
\frac{1}{n} \sum_{i=1}^{n} a(i)=\frac{1}{n} \sum_{p \leq n} p \geq \frac{1}{n} \sum_{n / 2 \leq p \leq n} p \geq \frac{1}{2}\left(\pi(n)-\pi\left(\frac{n}{2}\right)\right) \geq \frac{1}{2} .
$$

Démontrons maintenant le théorème 6 . Nous prenons $r(n)=n$ et nous utilisons le résultat élémentaire suivant [15] :

Lemme 1. Pour une infinité d'entiers $n$, on a

$$
p_{n+1}-p_{n} \geq 1.7 \log p_{n} .
$$

Il résulte immédiatement de ce lemme qu'il existe une suite de nombres premiers $n_{k}$ telle que

$$
a\left(n_{k}+1\right)=a\left(n_{k}+2\right)=\ldots=a\left(n_{k}+k\right)=0 \quad \text { avec } k \geq \frac{3}{2} \log n_{k} .
$$

On a donc $n_{k} \leq \exp \left(\frac{2}{3} k\right)$, et puisque $\exp \frac{2}{3}<2$, on a

$$
\lim _{k \rightarrow \infty} \frac{r\left(n_{k}+k+1\right)}{|q|^{k}}=0 .
$$

Donc si $x=\alpha / \beta \in \mathbb{Q}$, on a pour $k$ assez grand, en vertu du théorème 2 ,

$$
\alpha q^{n_{k}}-\beta \sum_{n=0}^{n_{k}} a(n) q^{n_{k}-n}=0 .
$$

Posons $\beta=q^{\gamma} \beta^{\prime}$, où $q$ ne divise par $\beta^{\prime}$. Alors

$$
\alpha q^{n_{k}-\gamma}-\beta^{\prime} \sum_{n=0}^{n_{k}} a(n) q^{n_{k}-n}=0 .
$$


On en déduit que $q$ divise $\beta^{\prime} a\left(n_{k}\right)=\beta^{\prime} n_{k}$. Puisque $n_{k}$ est premier, $q$ divise $\beta^{\prime}$, et cette contradiction démontre le théorème 6 .

\section{Un lemme sur les zéros de la suite $\varrho(n)$}

Lemme 2. Soit $\delta \in \mathbb{N}^{*}$, et soit $\left.a \in\right] 0,1\left[\right.$. On désigne par $p_{1}<p_{2}<\ldots<$ $p_{n}<\ldots$ la suite des nombres premiers de $\mathbb{N}$ qui sont congrus à 3 modulo 4 , $p_{1}$ étant choisi de façon que

$$
\sum_{n=1}^{\infty} \frac{1}{p_{n}^{2}} \leq \frac{a}{2}
$$

Alors il existe un entier $m_{0}=m_{0}(a, \delta)$ et une constante $L$ (constante de Linnik, voir théorème 5 ci-dessus) tels que, pour tout $k=p_{1} p_{2} \ldots p_{m}$, $m \geq m_{0}$, il existe $n_{k} \in \mathbb{N}$ tel que,

(a) $\varrho\left(n_{k}-\delta\right)=\ldots=\varrho\left(n_{k}-1\right)=\varrho\left(n_{k}+1\right)=\ldots=\varrho\left(n_{k}+k\right)=0$,

(b) $\varrho\left(n_{k}\right)=2$,

(c) $l\left(n_{k}-\delta\right)=\ldots=l\left(n_{k}\right)=\ldots=l\left(n_{k}+k\right)=0$,

(d) $n_{k} \leq 4^{L} \exp \left(4 L p_{2[a k]}\right)$.

Démonstration. Pour tout $x \in \mathbb{Z}$, et tout nombre premier $p$, nous notons $\nu_{p}(x)$ la valuation $p$-adique de $x$.

Pour $h \in \mathbb{N}^{*}$, soit $\mathbb{P}_{h}=\left\{p_{1}, p_{2}, \ldots, p_{h}\right\}$.

Pour $m \in \mathbb{N}^{*}$, notons $k=k(m)=p_{1} p_{2} \ldots p_{m}$.

Première étape. (a) Soit $\mathbb{M}_{m}=\left\{n \in\{1,2, \ldots, k\}: \exists p \in \mathbb{P}_{m}, \nu_{p}(n)>0\right\}$. En vertu de la formule du crible ([17], p. 22; [12], th. 261, p. 234),

$$
\operatorname{card} \mathbb{M}_{m}=\sum_{i}\left[\frac{k}{p_{i}}\right]-\sum_{i \neq j}\left[\frac{k}{p_{i} p_{j}}\right]+\ldots+(-1)^{m+1}\left[\frac{k}{p_{1} p_{2} \ldots p_{m}}\right] .
$$

Or $\nu_{p}(k)>0, \forall p \in \mathbb{P}_{m}$, donc

$$
\operatorname{card} \mathbb{M}_{m}=k-k\left(1-\frac{1}{p_{1}}\right)\left(1-\frac{1}{p_{2}}\right) \ldots\left(1-\frac{1}{p_{m}}\right) .
$$

(b) Soit $\mathbb{H}_{m}=\left\{n \in\{1,2, \ldots, k\}: \exists p \in \mathbb{P}_{m}, \nu_{p}(n) \geq 2\right\}$. On a

$$
\operatorname{card} \mathbb{H}_{m} \leq\left[\frac{k}{p_{1}^{2}}\right]+\left[\frac{k}{p_{2}^{2}}\right]+\ldots+\left[\frac{k}{p_{m}^{2}}\right] .
$$

En utilisant (12), on obtient

$$
\operatorname{card} \mathbb{H}_{m} \leq k \sum_{i=1}^{n} \frac{1}{p_{i}^{2}} \leq \frac{a}{2} k .
$$


(c) Soit $\mathbb{I}_{m}=\left\{n \in\{1,2, \ldots, k\}: \exists p \in \mathbb{P}_{m}, \nu_{p}(n)=1\right\}$. On a card $\mathbb{I}_{m}=$ $\operatorname{card} \mathbb{M}_{m}-\operatorname{card} \mathbb{H}_{m}$. Donc

$$
\operatorname{card} \mathbb{I}_{m} \geq k\left(1-\frac{a}{2}-\prod_{i=1}^{m}\left(1-\frac{1}{p_{i}}\right)\right) .
$$

Mais le produit infini $\prod_{i=1}^{\infty}\left(1-1 / p_{i}\right)$ diverge vers 0 car la série $\sum_{i=1}^{\infty} 1 / p_{i}$ diverge. Donc il existe un entier $m_{1}=m_{1}(a)$ tel que

$$
\forall m \geq m_{1}, \quad \operatorname{card} \mathbb{I}_{m} \geq k(1-a) .
$$

(d) Il en résulte que, pour $m \geq m_{1}$, tous les éléments de $\{1,2, \ldots, k\}$ sont divisibles par un facteur $p \in \mathbb{P}_{m}$ avec un exposant 1 exactement (et ne sont donc pas des sommes de deux carrés, [12], p. 299, th. 366), sauf $N=N(m)$ d'entre eux que nous noterons $u_{1}<u_{2}<\ldots<u_{N}$, et on a, en vertu de (14),

$$
\forall m \geq m_{1}, \quad N \leq a k .
$$

Deuxième étape. (a) Nous construisons maintenant $k=k(m)$ nombres entiers consécutifs $e_{m}+1, e_{m}+2, \ldots, e_{m}+k$ tels que

$$
\begin{aligned}
\forall m \in \mathbb{N}^{*}, \quad m \geq m_{1} \Rightarrow & \forall i \in\{1,2, \ldots, k\}, \\
& \exists p \in \mathbb{P}_{m+N}, \nu_{p}\left(e_{m}+i\right)=1 .
\end{aligned}
$$

On procède de la façon suivante : soit d'abord $r_{1} \in \mathbb{N}$ tel que

$$
\begin{gathered}
0 \leq r_{1}<2 p_{m+1}, \\
\nu_{p_{m+1}}\left(r_{1}\left(p_{1} p_{2} \ldots p_{m}\right)^{2}+u_{1}\right)=1 .
\end{gathered}
$$

Puis soit $r_{h}(1 \leq h \leq N)$ défini par récurrence par

$$
\begin{gathered}
0 \leq r_{h}<2 p_{m+h}, \\
\nu_{p_{m+h}}\left(r_{h}\left(p_{1} p_{2} \ldots p_{m+h-1}\right)^{2}+r_{h-1}\left(p_{1} p_{2} \ldots p_{m+h-2}\right)^{2}+\ldots\right. \\
\left.+r_{1}\left(p_{1} p_{2} \ldots p_{m}\right)^{2}+u_{h}\right)=1 .
\end{gathered}
$$

Nous posons

$$
\begin{array}{r}
e_{m}=r_{N}\left(p_{1} p_{2} \ldots p_{m+N-1}\right)^{2}+r_{N-1}\left(p_{1} p_{2} \ldots p_{m+N-2}\right)^{2}+\ldots \\
+r_{1}\left(p_{1} p_{2} \ldots p_{m}\right)^{2} .
\end{array}
$$

Soit $i \in\{1,2, \ldots, k\}$. Il est clair par construction que, si $j \in\{1,2, \ldots$ $\ldots, m\}, e_{m}+i \equiv i\left(\bmod p_{j}^{2}\right)$. Donc si $i \in \mathbb{I}_{m}$, il existe $p \in \mathbb{P}_{m}$ tel que $\nu_{p}\left(e_{m}+i\right)=1$. Si $i \notin \mathbb{I}_{m}$, il existe $h \in\{1,2, \ldots, N\}$ tel que $i=u_{h}$; on a alors

$$
e_{m}+i \equiv r_{h}\left(p_{1} p_{2} \ldots p_{m+h-1}\right)^{2}+\ldots+r_{1}\left(p_{1} p_{2} \ldots p_{m}\right)^{2}+u_{h}\left(\bmod p_{m+h}^{2}\right),
$$

donc $\nu_{p_{m+h}}\left(e_{m}+i\right)=1$ en vertu de (18).

Par conséquent, le nombre $e_{m}$ défini par (19) vérifie bien (16). 
(b) On peut majorer $e_{m}$, puisque $r_{h}<2 p_{m+h}, \forall h \in\{1,2, \ldots, N\}$ :

$$
\begin{aligned}
e_{m} \leq & 2 p_{m+N}\left(p_{1} p_{2} \ldots p_{m+N-1}\right)^{2} \\
& +2 p_{m+N-1}\left(p_{1} p_{2} \ldots p_{m+N-2}\right)^{2}+\ldots+2 p_{m+1}\left(p_{1} p_{2} \ldots p_{m}\right)^{2}, \\
e_{m} \leq & 2\left(p_{1} p_{2} \ldots p_{m+N}\right)^{2} \\
& \times\left(\frac{1}{p_{m+N}}+\frac{1}{p_{m+N-1} p_{m+N}^{2}}+\ldots+\frac{1}{p_{m+1} p_{m+2}^{2} \ldots p_{m+N}^{2}}\right), \\
e_{m} \leq & 2\left(p_{1} p_{2} \ldots p_{m+N}\right)^{2}\left(\frac{1}{3}+\frac{1}{3 \cdot 3^{2}}+\ldots+\frac{1}{3 \cdot 3^{2 N-2}}\right) .
\end{aligned}
$$

Donc

$$
e_{m} \leq\left(p_{1} p_{2} \ldots p_{m+N}\right)^{2}
$$

Troisième étape. Soit maintenant

$$
\mathbb{J}_{m}=\left\{j \in \mathbb{N}: \forall i \in\{1,2, \ldots, k\}, \exists p \in \mathbb{P}_{m+N}, \nu_{p}(j+i)=1\right\} .
$$

$\mathbb{J}_{m}$ est non vide puisqu'il contient $e_{m}$. Soit $q_{m}$ le plus petit élément de $\mathbb{J}_{m}$. On a alors

$$
\begin{gathered}
q_{m} \leq\left(p_{1} p_{2} \ldots p_{m+N}\right)^{2}, \\
\forall p \in \mathbb{P}_{m+N}, \quad \nu_{p}\left(q_{m}\right) \neq 1 .
\end{gathered}
$$

Quatrième étape. (a) Notons $v_{1}<v_{2}<\ldots<v_{M}$ les éléments de $\{1,2, \ldots, \delta\}$ tels que $\forall j \in\{1,2, \ldots, M\}, \forall p \in \mathbb{P}_{m+N}, \nu_{p}\left(q_{m}-v_{j}\right) \neq 1$.

On a donc $M \leq \delta$.

De même que dans l'étape 2 , nous construisons la suite $r_{i}^{\prime}$ par les relations suivantes :

$$
\begin{gathered}
0 \leq r_{1}^{\prime}<2 p_{m+N+1}, \\
\nu_{p_{m+N+1}}\left(r_{1}^{\prime}\left(p_{1} p_{2} \ldots p_{m+N}\right)^{2}+q_{m}-v_{1}\right)=1 .
\end{gathered}
$$

Puis par récurrence, pour $1 \leq h \leq M-1$, nous posons

$$
\begin{gathered}
0 \leq r_{h}^{\prime}<2 p_{m+N+h} \\
\nu_{p_{m+N+h}}\left(r_{h}^{\prime}\left(p_{1} p_{2} \ldots p_{m+N+h-1}\right)^{2}+r_{h-1}^{\prime}\left(p_{1} p_{2} \ldots p_{m+N+h-2}\right)^{2}\right. \\
\left.+\ldots+r_{1}^{\prime}\left(p_{1} p_{2} \ldots p_{m+N}\right)^{2}+q_{m}-v_{h}\right)=1 .
\end{gathered}
$$

Nous imposons à $r_{M}^{\prime}$ des conditions supplémentaires; pour cela, nous notons $\mathbb{L}_{m+N}=\left\{p \in \mathbb{P}_{m+N}: \nu_{p}\left(q_{m}\right)=2\right\}$.

Soit $T$ vérifiant le système de congruences

$$
\begin{aligned}
T\left(p_{1} p_{2}\right. & \left.\ldots p_{m+N+M-1}\right)^{2}+r_{M-1}^{\prime}\left(p_{1} p_{2} \ldots p_{m+N+M-2}\right)^{2}+\ldots \\
& +r_{1}^{\prime}\left(p_{1} p_{2} \ldots p_{m+N}\right)^{2}+q_{m}-v_{M} \equiv 0\left(\bmod p_{m+N+M}\right),
\end{aligned}
$$




$$
\begin{array}{r}
T\left(p_{m+N+1} p_{m+N+2} \ldots p_{m+N+M-1}\right)^{2} \\
+r_{M-1}^{\prime}\left(p_{m+N+1} \ldots p_{m+N+M-2}\right)^{2}+\ldots+r_{1}^{\prime} \equiv 0(\bmod p) \\
\text { si } p \in \mathbb{L}_{m+N}, \\
T\left(p_{m+N+1} p_{m+N+2} \ldots p_{m+N+M-1}\right)^{2} \\
+r_{M-1}^{\prime}\left(p_{m+N+1} \ldots p_{m+N+M-2}\right)^{2}+\ldots+r_{1}^{\prime} \equiv 1(\bmod p) \\
\text { si } p \in \mathbb{P}_{m+N} \backslash \mathbb{L}_{m+N} .
\end{array}
$$

On sait ([12], th. 121, p. 95) que l'on peut prendre $T$ solution de ce système, et vérifiant

$$
0 \leq T \leq p_{1} p_{2} \ldots p_{m+N} p_{m+N+M} .
$$

Soit $H=T\left(p_{1} p_{2} \ldots p_{m+N+M-1}\right)^{2}+r_{M-1}^{\prime}\left(p_{1} p_{2} \ldots p_{m+N+M-2}\right)^{2}+\ldots+$ $r_{1}^{\prime}\left(p_{1} p_{2} \ldots p_{m+N}\right)^{2}+q_{m}-v_{M}$ (voir congruence $\left.(24)\right)$.

Nous posons $r_{M}^{\prime}=T$ si $H$ n'est pas un multiple de $p_{m+N+M}^{2}$, et $r_{M}^{\prime}=$ $T+p_{1} p_{2} \ldots p_{m+N} p_{m+N+M}$ si $H$ est un multiple de $p_{m+N+M}^{2}$.

Il est clair alors que $r_{M}^{\prime}$ vérifie les conditions suivantes :

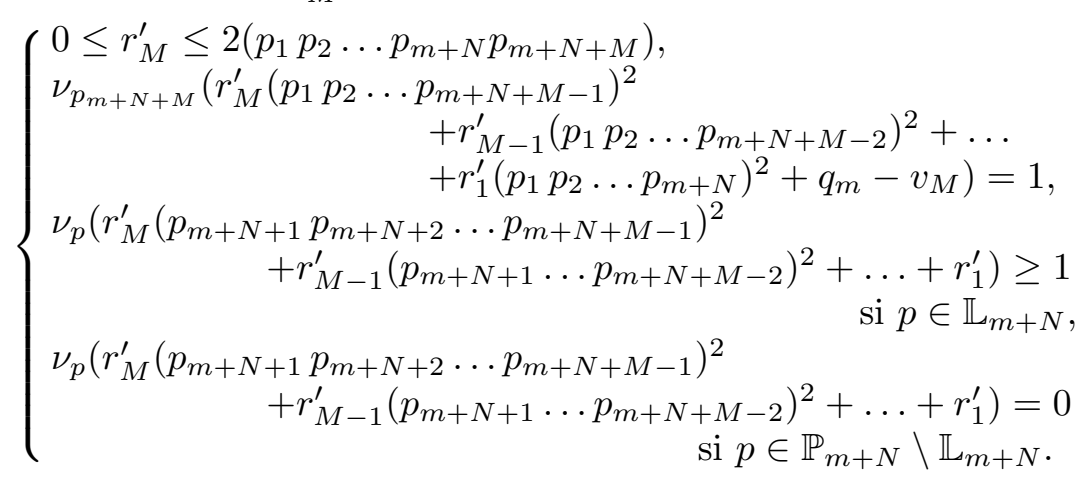

(b) Posons maintenant

$$
\begin{aligned}
t_{m}=r_{M}^{\prime}\left(p_{1} p_{2} \ldots p_{m+N+M-1}\right)^{2} & +r_{M-1}^{\prime}\left(p_{1} p_{2} \ldots p_{m+N+M-2}\right)^{2} \\
& +\ldots+r_{1}^{\prime}\left(p_{1} p_{2} \ldots p_{m+N}\right)^{2}+q_{m} .
\end{aligned}
$$

Par construction,

$$
\begin{gathered}
\forall i \in\{-\delta,-\delta+1, \ldots,-1,1,2, \ldots, k\}, \exists p \in \mathbb{P}_{m+N+M}, \text { tel que : } \\
\nu_{p}\left(t_{m}+i\right)=1 .
\end{gathered}
$$

(c) Par ailleurs, en vertu de l'avant-dernière condition de (27) : $\forall p \in$ $\mathbb{L}_{m+N}, \nu_{p}\left(t_{m}\right)=2$, car on a alors $\nu_{p}\left(q_{m}\right)=2$ et $\nu_{p}\left(t_{m}-q_{m}\right) \geq 3$.

Si $p \in \mathbb{P}_{m+N} \backslash \mathbb{L}_{m+N}$, deux cas peuvent se produire car $\nu_{p}\left(q_{m}\right) \neq 1$ grâce à $(21)$ :

( $\alpha) \nu_{p}\left(q_{m}\right)=0$, auquel cas $\nu_{p}\left(t_{m}\right)=0$.

( $\beta) \nu_{p}\left(q_{m}\right) \geq 3$; alors $\nu_{p}\left(t_{m}\right)=2$ à cause de la dernière condition de $(27)$ qui implique $\nu_{p}\left(t_{m}-q_{m}\right)=2$. 
On a donc $\forall p \in \mathbb{P}_{m+N}, \nu_{p}\left(t_{m}\right)=0$ ou 2 .

De plus, $\forall m \geq \delta, \forall i \in\{m+N+1, \ldots, m+N+M\}, \nu_{p_{i}}\left(t_{m}\right)=0$ car il existe un entier $j, 1 \leq j \leq M$, tel que $\nu_{p_{i}}\left(t_{m}-v_{j}\right)=1$.

Finalement, nous avons donc :

(30) Pour $m$ assez grand, $\forall p \in \mathbb{P}_{m+N+M}, \nu_{p}\left(t_{m}\right)=0$ ou 2 .

(d) Nous majorons maintenant $t_{m}$ à partir de (21), (22), (23), (27) :

$$
\begin{aligned}
t_{m} \leq & 2\left(p_{1} p_{2} \ldots p_{m+N} p_{m+N+M}\right)\left(p_{1} p_{2} \ldots p_{m+N+M-1}\right)^{2} \\
& +2 p_{m+N+M-1}\left(p_{1} p_{2} \ldots p_{m+N+M-2}\right)^{2}+\ldots \\
& +2 p_{m+N+1}\left(p_{1} p_{2} \ldots p_{m+N}\right)^{2}+\left(p_{1} p_{2} \ldots p_{m+N}\right)^{2} .
\end{aligned}
$$

Donc

$$
t_{m}=o\left(\left(p_{1} p_{2} \ldots p_{m+N+M}\right)^{4}\right) .
$$

Cinquième étape. (a) Soit maintenant $\eta=\eta(m) \in\{0,1,2,3\}$ tel que

$$
\eta\left(p_{1} p_{2} \ldots p_{m+N+M}\right)^{4}+t_{m} \equiv 1(\bmod 4) .
$$

Pour $s \in \mathbb{N}$, considérons

$$
w_{s}=4\left(p_{1} p_{2} \ldots p_{m+N+M}\right)^{4} s+\eta\left(p_{1} p_{2} \ldots p_{m+N+M}\right)^{4}+t_{m} .
$$

On a d'abord

$$
\forall s \in \mathbb{N}, \quad w_{s} \equiv 1(\bmod 4) .
$$

De plus, en vertu de (29),

$$
\begin{aligned}
\forall s \in \mathbb{N}, \forall i \in\{-\delta,-\delta+1, \ldots, & -1,1,2, \ldots, k\}, \\
& \exists p \in \mathbb{P}_{m+N+M}, \quad \nu_{p}\left(w_{s}+i\right)=1 .
\end{aligned}
$$

(b) Soit $D$ le plus grand diviseur commun à $4\left(p_{1} p_{2} \ldots p_{m+N+M}\right)^{4}$ et $\eta\left(p_{1} p_{2} \ldots p_{m+N+M}\right)^{4}+t_{m}$. En utilisant (30) et (32), on voit que, pour $m$ assez grand,

$$
D=\prod_{i=1}^{m+N+M} p_{i}^{\alpha_{i}}, \quad \text { avec } \alpha_{i}=0 \text { ou } 2 .
$$

(c) Posons $w_{s}=D(\zeta s+\chi)$, avec $(\zeta, \chi) \in \mathbb{N} \times \mathbb{N}$. En vertu de (31), puisque $\eta \in\{0,1,2,3\}$, on a $\chi<\zeta$ pour $m$ assez grand. On peut alors utiliser le théorème 5 : il existe un nombre premier $p(\zeta, \chi)$ et un entier naturel $\sigma$ tels que

$$
w_{\sigma}=D \cdot p(\zeta, \chi) \leq D\left(\frac{4\left(p_{1} p_{2} \ldots p_{m+N+M}\right)^{4}}{D}\right)^{L} .
$$

Nous posons

$$
n_{k}=n_{k(m)}=w_{\sigma} .
$$


Nous avons donc, puisque $L \geq 1$ :

$$
n_{k} \leq\left(4\left(p_{1} p_{2} \ldots p_{m+N+M}\right)^{4}\right)^{L} .
$$

Par ailleurs, $D \equiv 1(\bmod 4)$ d'après $(36)$, et $n_{k} \equiv 1(\bmod 4)$ d'après (34) et $(38)$. Ainsi $p(\zeta, \chi) \equiv 1(\bmod 4)$, d'où l'on déduit que $p(\zeta, \chi)$ est une somme de deux carrés. De plus, puisque la décomposition de $D$ ne contient que des facteurs premiers qui ne sont pas somme de deux carrés (voir (36)), on a

$$
\varrho\left(n_{k}\right)=\varrho[p(\zeta, \chi)]=2
$$

(voir [12], formule (16.9.5) et th. 278, p. 242).

Si nous regroupons (35) et (40), nous obtenons les conclusions (a), (b) et (c) du lemme 2 . Il reste à démontrer la majoration $(\mathrm{d})$.

Sixième (et dernière) étape. (a) En utilisant le théorème des nombres premiers dans les suites arithmétiques ([9], p. 70), on obtient

$$
m+N+M \sim \frac{1}{2} \frac{p_{m+N+M}}{\log p_{m+N+M}} \quad \text { lorsque } m \rightarrow \infty .
$$

Donc

$$
m+N+M \leq \frac{p_{m+N+M}}{\log p_{m+N+M}} \quad \text { pour } m \geq m_{2} .
$$

Utilisant (39) et (42), nous obtenons

$$
n_{k} \leq 4^{L}\left(p_{m+N+M}\right)^{4 L(m+N+M)} \leq 4^{L} \exp \left(4 L p_{m+N+M}\right) .
$$

(b) Mais on a aussi $k=p_{1} p_{2} \ldots p_{m} \geq p_{1}^{m}$, donc

$$
m \leq \frac{\log k}{\log p_{1}} \text {. }
$$

Et puisque $M \leq \delta$, utilisant (15) et (44), nous obtenons

si bien que, pour $m \geq m_{3}$,

$$
m+N+M \leq \frac{\log k}{\log p_{1}}+a k+\delta,
$$

$$
m+N+M \leq 2[a k] .
$$

Reportons ce résultat dans (43); nous obtenons

$$
n_{k} \leq 4^{L} \exp \left(4 L p_{2[a k]}\right),
$$

ce qui achève la démonstration du lemme 2.

5. Démonstration du théorème 1. Supposons qu'il existe $A, B, C \in$ $\mathbb{Z}, A \neq 0$, tels que $A x_{q}^{2}+B x_{q}+C=0$.

Nous allons utiliser le théorème 2 avec $a(n)=A \varrho(n)+B l(n)$, et montrer que nous aboutissons à une contradiction. 
(a) Puisque $A \neq 0$, nous pouvons choisir $\delta$ tel que $q^{\delta}$ ne divise pas $2 A$. Nous choisissons de plus $a \in] 0,1[$ tel que

$$
32 L-\frac{\log |q|}{8 a}<0 .
$$

(b) Majorons maintenant $|a(n)| \leq|A| \varrho(n)+|B|$. On sait que

$$
\varrho(n) \leq d(n) \leq \exp \left(\frac{\log n}{\log \log n}\right)
$$

pour $n$ assez grand ([12], p. 262, th. 317 et $\S 18-7$, p. 270). Nous pouvons donc écrire $|a(n)| \leq r(n), \forall n \geq n_{0}$ avec

$$
r(n)=\exp \left(\frac{2 \log n}{\log \log n}\right) .
$$

(c) Nous appliquons le lemme 2, $a$ et $\delta$ étant définis ci-dessus. On a

$$
n_{k}+k+1 \leq 4^{L} \exp \left(4 L p_{2[a k]}\right)+k+1 .
$$

Or, en utilisant le théorème des nombres premiers dans les suites arithmétiques, on a, pour $k$ assez grand,

$$
\frac{1}{4} \frac{p_{2[a k]}}{\log p_{2[a k]}} \leq 2 a k \leq \frac{p_{2[a k]}}{\log p_{2[a k]}} .
$$

Pour $k$ assez grand, (49) entraîne donc

$$
n_{k}+k+1 \leq \exp \left(8 L p_{2[a k]}\right) \text {. }
$$

La fonction $x \mapsto \log x / \log \log x$ étant croissante pour $x \geq e^{e}$, on obtient

$$
\log r\left(n_{k}+k+1\right)=\frac{2 \log \left(n_{k}+k+1\right)}{\log \log \left(n_{k}+k+1\right)} \leq \frac{16 L p_{2[a k]}}{\log 8 L+\log p_{2[a k]}} .
$$

Donc, pour $k$ assez grand,

$$
r\left(n_{k}+k+1\right) \leq \exp \left(32 L \frac{p_{2[a k]}}{\log p_{2[a k]}}\right) .
$$

(d) Posons $x=p_{2[a k]} / \log p_{2[a k]}$. Alors $x$ tend vers l'infini avec $k$. En utilisant (50) et (51), on obtient

$$
\frac{r\left(n_{k}+k+1\right)}{\mid q^{k}} \leq \frac{\exp (32 L x)}{\mid q^{\mid x /(8 a)}},
$$

d'où

$$
\frac{r\left(n_{k}+k+1\right)}{|q|^{k}} \leq \exp \left(\left(32 L-\frac{\log |q|}{8 a}\right) x\right)
$$

et puisque $a$ vérifie (46), $\lim _{k \rightarrow \infty} r\left(n_{k}+k+1\right) /|q|^{k}=0$.

(e) Il est clair par ailleurs que $\lim _{n \rightarrow \infty} r(n+1) / r(n)=1$. Comme $a\left(n_{k}+1\right)=a\left(n_{k}+2\right)=\ldots=a\left(n_{k}+k\right)$ en vertu du lemme 2 , le théorème 
2 s'applique, et on obtient, pour $k=k(m)$ assez grand,

$$
C q^{n_{k}}-\sum_{n=0}^{n_{k}} a(n) q^{n_{k}-n}=0 .
$$

Utilisant les (a), (b) et (c) du lemme 2, on obtient

$$
C q^{n_{k}}-2 A-\sum_{n=0}^{n_{k}-\delta-1} a(n) q^{n_{k}-n}=0 .
$$

On en conclut que $q^{\delta+1}$ divise $2 A$, ce qui contredit le choix de $\delta$. Le théorème 1 est donc démontré.

\section{Bibliographie}

[1] P. Borwein, On the irrationality of certain series, Math. Proc. Cambridge Philos. Soc. 112 (1992), 141-146.

[2] P. Bundschuh, Verschärfung eines arithmetischen Satzes von Tschakaloff, Portugal. Math. 33 (1) (1974), 1-47.

[3] —, Quelques résultats arithmétiques sur les fonctions thêta de Jacobi, Publications mathématiques de l'Université Pierre et Marie Curie no. 64, Fasc. 1, Groupe d'étude sur les problèmes diophantiens, 1983/84.

[4] P. Bundschuh and M. Waldschmidt, Irrationality results for theta functions by Gel'fond-Schneider's method, Acta Arith. 53 (1989), 289-307.

[5] K. Chandrasekharan, Elliptic Functions, Grundlehren Math. Wiss. 281, Springer, 1985.

[6] P. Erdős, Sur l'irrationalité d'une certaine série, C. R. Acad. Sci. Paris Sér. I 292 (1981), 765-768.

[7] - On the irrationality of certain series: problems and results, dans : New Advances in Transcendence Theory, A. Baker (ed.), Cambridge University Press, 1988, 102109.

[8] H. Exton, q-Hypergeometric Functions and Applications, Ellis Horwood, Chichester 1983.

[9] A. Gel'fond et Y. Linnik, Méthodes élémentaires dans la théorie analytique des nombres, Gauthier-Villars, 1965.

[10] S. Golomb, A new arithmetic function of combinatorial significance, J. Number Theory 5 (1973), 218-223.

[11] S. Graham, On Linnik's constant, Acta Arith. 39 (1981), 163-179.

[12] G. H. Hardy and E. M. Wright, An Introduction to the Theory of Numbers, Oxford Sci. Publ., Oxford Univ. Press, 1989.

[13] Yu. V. Linnik, Sur le plus petit nombre premier dans une progression arithmétique, Mat. Sb. 15 (57) (1944), 139-178 (en russe).

[14] J. Liouville, Sur des classes très étendues de quantités dont la valeur n'est ni algébrique ni même réductible à des irrationnelles algébriques, J. Math. Pures Appl. (1) 16 (1851), 133-142.

[15] B. Powell and E. Schafer, Difference between consecutive primes, Amer. Math. Monthly 91 (1984), 310-311.

[16] P. Ribenboim, The Book of Prime Numbers Records, Springer, 1984. 
[17] H. J. Ryser, Mathématiques combinatoires, Dunod, 1969.

[18] L. Tschakaloff, Arithmetische Eigenschaften der unendlichen Reihe $\sum_{\nu=0}^{\infty} x^{\nu} a^{-\nu(\nu-1) / 2}$, Math. Ann. 80 (1921), 62-74.

24 PLACE DU CONCERT

F-59800 LILLE, FRANCE

Reçu le 19.6.1992

et révisé le 1.12.1992 